\title{
DOS ESPECIES NUEVAS DE COMPOSITAE, EUPATORIEAE DE MÉXICO*
}

\author{
Jerzy Rzedowski ${ }^{1}$ y Graciela Calderón de Rzedowski \\ Instituto de Ecología, A.C., Centro Regional del Bajío, \\ Apdo. postal 386, 61600 Pátzcuaro, Michoacán, México. \\ ${ }^{1}$ Autor para la correspondencia: jerzy.rzedowski@inecol.edu.mx
}

\begin{abstract}
RESUMEN
Se describen como nuevas e ilustran dos especies mexicanas de CompositaeEupatorieae. Brickellia leonis, de Michoacán, pertenece al grupo anteriormente separado como género Kuhnia en virtud de sus cerdas plumosas del vilano y parece estar relacionada con B. adenolepis (B. L. Rob.) Shinners. Oxylobus juarezensis, conocido de una sola colecta de la Sierra Juárez de Oaxaca, se vincula más cercanamente con O. oaxacanus Blake.

Palabras clave: Brickellia, Compositae, México, Oxylobus.
\end{abstract}

\begin{abstract}
Two new Mexican species of Compositae-Eupatorieae are described and illustrated. Brickellia leonis, from Michoacán, belongs to the group formerly recognized as a separate genus Kuhnia, in accordance with its plumose pappus bristles, and seems to be related to B. adenolepis (B. L. Rob.) Shinners. Oxylobus juarezensis, known from a single collection from the Sierra de Juárez of Oaxaca, is more closely related to O. oaxacanus Blake.
\end{abstract}

Kew words: Brickellia, Compositae, Mexico, Oxylobus.

Como resultado de trabajo de revisión de materiales acumulados en el herbario de Pátzcuaro, se dan a conocer las siguientes novedades.

* Trabajo realizado con apoyo económico del Instituto de Ecología, A.C., del Consejo Nacional de Ciencia y Tecnología y de la Comisión Nacional para el Conocimiento y Uso de la Biodiversidad. 
Brickellia leonis Rzedowski \& Calderón sp. n. (Fig. 1)

Herba perennis $15(25) \mathrm{cm}$ alta; rami decumbentes vel ascendentes puberuli; folia alterna vel aliqua opposita, breviter petiolata, laminis anguste oblongis vel ellipticis $0.8-1.4(1.7) \mathrm{cm}$ longis integris ad basem attenuatis trinervatis vel triplinervatis, supra scabridis, infra glabris; pedunculi monocephali usque ad $20 \mathrm{~cm}$ longi; capitulum turbinatum 1.5-2 cm longum, involucro graduato in (2)3(4) seriebus, phylariis 16-20 oblongis ad lanceolatis vel ovatis usque ad $12 \mathrm{~mm}$ longis minute denseque puberulis, receptaculo plano, nudo; flores 20-23 per capitulum; corollae anguste cylindricae 8-9 $\mathrm{mm}$ longae vinaceae, lobulis minus quam $1 \mathrm{~mm}$ longis; styli lobi claviformes; achaenia lineari-claviformia leviter compressa 6-7 mm longa brunnea, 10-12 nervis longitudinalibus, pilosula, pappus 35 setis inaequalibuis plumosis 5-7 $\mathrm{mm}$ longis brunneo-albidis.

Planta herbácea perenne de 15(25) cm de alto; porciones subterráneas en forma de base rizomatosa muy engrosada, tuberiforme, algo leñosa, de dirección vertical, de la cual parten varias o numerosas raíces, así como uno o con más frecuencia algunos tallos que llevan un corto trayecto subterráneo y al salir a la superficie se dividen en varias ramas decumbentes o ascendentes de disposición radial y comúnmente un escapo vertical que lleva una sola cabezuela terminal; tallos pubérulos con pelos aplicados de 0.1 a $0.2 \mathrm{~mm}$ de largo; hojas alternas o a veces algunas opuestas, peciolo de (0)1 a 1.5(2) mm de largo, lámina estrechamente oblonga o elíptica, de 0.8 a 1.4(1.7) $\mathrm{cm}$ de largo y de (1.5)2 a 3(5) mm de ancho, ápice agudo u obtuso, base atenuada y a veces decurrente sobre el peciolo, margen entero, trinervada o triplinervada y sin otra nervadura evidente, de textura membranácea, haz diminutamente escábrido, envés glabro, densamente cubierto con numerosas glándulas punctiformes; pedúnculo monocéfalo, hasta de $20 \mathrm{~cm}$ de largo, con frecuencia morado, glabro o casi glabro en la parte inferior a densamente pubérulo por lo general en la superior, llevando 1 a 4 brácteas lineares a lanceoladas, de 4 a 5(6) $\mathrm{mm}$ de largo; cabezuela turbinada, de 1.5 a $2 \mathrm{~cm}$ de largo; involucro graduado en (2)3(4) series, sus brácteas 16 a 20, oblongas a lanceoladas u ovadas, las más largas de ca. $12 \mathrm{~mm}$ de longitud, todas o casi todas de ca. $2 \mathrm{~mm}$ de ancho, agudas, acuminadas o mucronadas en el ápice, con frecuencia moradas en la parte distal, diminuta y densamente pubérulas, receptáculo plano, desnudo, alveolado; flores 20 a 23 por cabezuela; corolas angostamente cilíndricas, de 8 a $9 \mathrm{~mm}$ de largo y menos de $1 \mathrm{~mm}$ de diámetro, de color guinda al menos en su parte distal, lóbulos de ca. $1 \mathrm{~mm}$ de largo; anteras de ca. $2.5 \mathrm{~mm}$ de largo, incluyendo el corto apéndice apical, sin sobresalir de la corola, coherentes en la juventud, separadas en la madurez, blanquecinas, transparentes; estilo de ca. $8 \mathrm{~mm}$ de largo, dividido 
Rzedowski y Calderón de Rzedowski: Dos especies nuevas de Eupatorieae de México

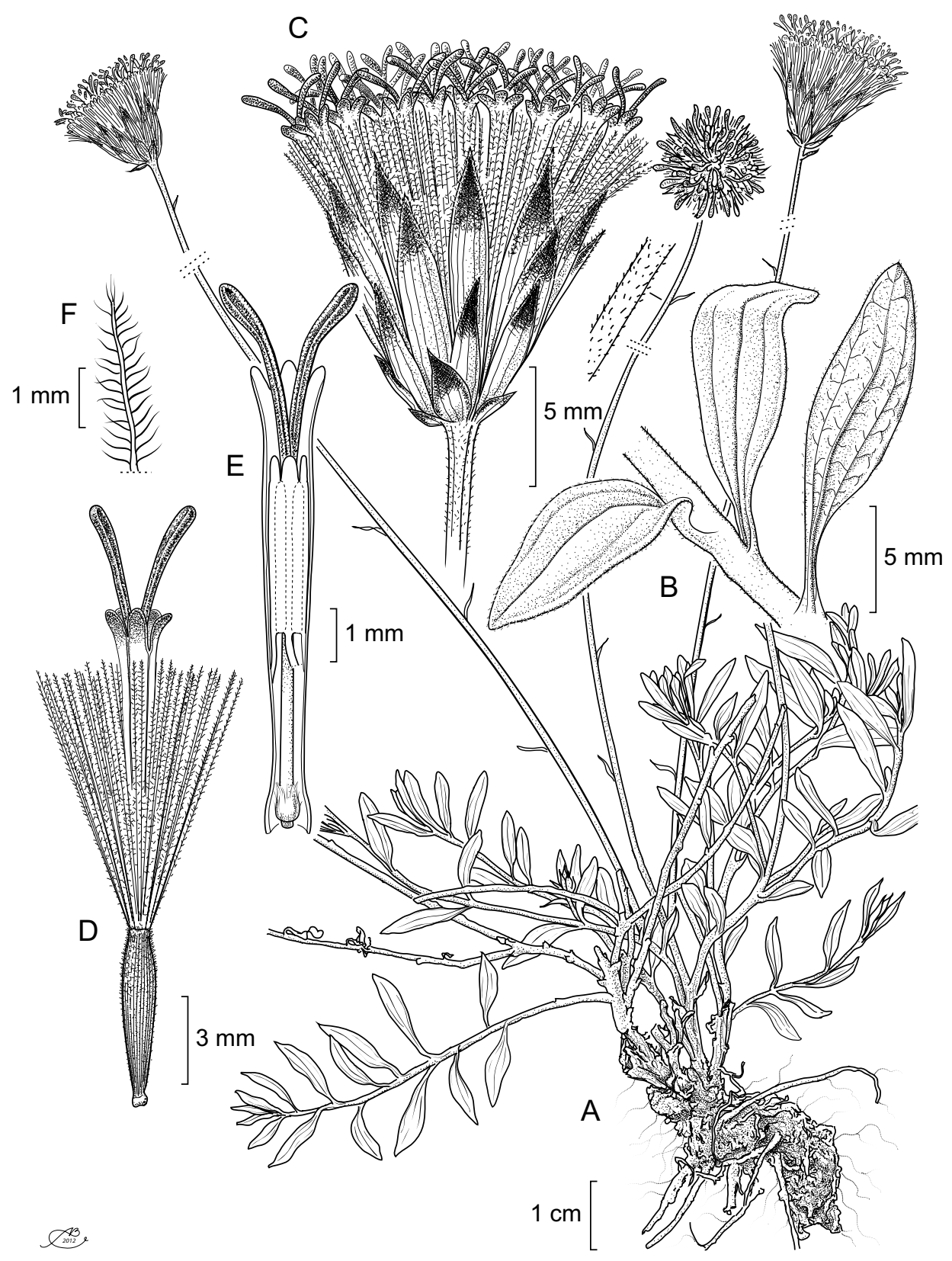

Fig. 1. Brickellia leonis Rzed. \& Calderón. A. aspecto general de la planta; B. hojas; C. cabezuela; D. flor completa con aquenio; E. disección de la corola para mostrar las anteras y el estilo; F. cerda del vilano. Ilustrado por Alfonso Barbosa. 
en cerca de la mitad de su largo, sus lóbulos claviformes, redondeados en el ápice y exsertos de la corola, de color guinda, en la base dotado de un disco grueso y lanoso; aquenios linear-claviformes, algo comprimidos, de 6 a $7 \mathrm{~mm}$ de largo, de color café, con 10 a 12 nervios longitudinales, pilósulos sobre todo en la mitad distal, vilano de ca. 35 cerdas plumosas desiguales, de 5 a $7 \mathrm{~mm}$ de largo, café-blanquecinas.

Tipo: México, Michoacán, cerca de Fontezuelas, municipio de Lagunillas, bosque de encino y claros adyacentes, alt. $2100 \mathrm{~m}$, 2.VI.1990, J. Rzedowski 49357 (IEB, isotipos por distribuirse).

Material adicional examinado: México, Michoacán, rancho Ziranga, sobre la carretera a Ihuatzio, municipio de Tzintzuntzan, pastizal secundario, alt. $2100 \mathrm{~m}$, 24.VII.1986, H. Díaz B. y N. López 2335 (IEB).

En virtud de su vilano de cerdas plumosas $B$. leonis está cercanamente emparentada con el grupo de B. eupatorioides (L.) Shinners, plantas que en otros tiempos se acostumbraban ubicar en el género separado Kuhnia.

Este conjunto fue estudiado por Shinners $(1946,1971)$ y posteriormente por Turner (1989), quien reconoció la existencia de tres especies: Brickellia adenolepis (B. L. Rob.) Shinners, B. oreithales (B. L. Rob.) Shinners y B. eupatorioides (L.) Shinners. Esta última con seis variedades, de las cuales la única presente en México es B. eupatorioides var. chlorolepis (Woot. \& Standl.) B. L. Turner.

En virtud de su porte escapiforme, $B$. leonis parece ubicarse más cerca de $B$. adenolepis; las principales diferencias entre estos dos taxa se resumen en el siguiente cuadro:

\begin{tabular}{lll}
\hline & B. adenolepis & B. leonis \\
\hline Cabezuelas & agrupadas en panículas & solitarias \\
$\begin{array}{l}\text { pubescencia de brácteas } \\
\text { involucrales }\end{array}$ & $\begin{array}{l}\text { con frecuencia con pelos } \\
\text { glandulosos }\end{array}$ & sin pelos glandulosos \\
ancho de brácteas involucrales & ca. $1 \mathrm{~mm}$ & ca. $2 \mathrm{~mm}$ \\
largo de la corola & 7 a $8 \mathrm{~mm}$ & 8 a $9 \mathrm{~mm}$ \\
largo del aquenio & ca. $5 \mathrm{~mm}$ & 6 a $7 \mathrm{~mm}$ \\
\hline
\end{tabular}

El epíteto de la especie conmemora el apellido del médico y botánico mexicano, oriundo de Michoacán, Nicolás León (1859-1929). Su obra más renombrada fue el libro intitulado "Biblioteca botánico-mexicana", publicado en 1895. 
Oxylobus juarezensis Rzedowski \& Calderón sp. n. (Fig. 2)

Frutex parvus usque $25 \mathrm{~cm}$ altus ramis foliisque in altitudine quam $10 \mathrm{~cm}$ minore aggregatis; folia opposita petiolis $4-12 \mathrm{~mm}$ longis, laminis ellipticis, ovatis vel oblongis, 0.7-2.5 cm longis dentatis vel crenatis, ad basem longe cuneatis, glabris; inflorescentiae terminales laxe subcorymbosae; involucrum 3.5-5 mm longum et latum, phylariis 8-10 glabris; flores per capitulum 12-15, corollae albidae ca. $4 \mathrm{~mm}$ longae, in floribus perifericis lobis leviter inaequalibus ca. $1.3 \mathrm{~mm}$ longis, in floribus centralibus lobis aequalibus ca. $0.8 \mathrm{~mm}$ longis; achaenia linearía vel fusiformia 3-4 mm longa, pappus cyathiformis $15-25$ lobis squamiformibus coronatus.

Arbusto pequeño hasta de $25 \mathrm{~cm}$ de alto, con las ramas y el follaje concentrados en menos de $10 \mathrm{~cm}$ de alto y $20 \mathrm{~cm}$ de diámetro; ramas con frecuencia postradas, glabras, las más tiernas a menudo moradas, entrenudos por lo general de menos de $2 \mathrm{~cm}$ de largo; hojas opuestas, peciolo de 4 a $12 \mathrm{~mm}$ de largo, glabro, lámina por lo común elíptica, variando a veces a ovada u oblonga, de 0.7 a $2.5 \mathrm{~cm}$ de largo, de 3 a $8(12) \mathrm{mm}$ de ancho, redondeada a aguda en el ápice, largamente cuneada en la base, dentada o crenada en el margen, triplinervada, de textura membranácea, glabra en ambas superficies; inflorescencias terminales, paniculiforme-subcorimbosas, pedúndulos primarios de 7 a $12 \mathrm{~cm}$ de largo, los secundarios y terciarios de 1 a $6 \mathrm{~cm}$ de largo, glabros, cabezuelas por inflorescencia 2 a 5(12); involucro campanulado, de 3.5 a $5 \mathrm{~mm}$ de largo y de diámetro, sus brácteas 8 a 10, ovadas u oblongas, todas aproximadamente del mismo largo, las exteriores por lo general más anchas que las interiores, todas o casi todas romas en el ápice, glabras, receptáculo plano, flores por cabezuela 12 a 15, sus corolas no todas iguales, angostamente infundibuliformes, blanquecinas, glabras, de ca. $4 \mathrm{~mm}$ de largo, del cual ca. $1.5 \mathrm{~mm}$ corresponde al tubo, otro tanto a la garganta y ca. $1 \mathrm{~mm}$ a los lóbulos, estos últimos son más largos (ca. $1.3 \mathrm{~mm}$ ) y ligeramente desiguales en la perifierie, y más cortos (ca. $0.8 \mathrm{~mm}$ ) y todos similares en el centro del disco; aquenios lineares a fusiformes, de 3 a $4 \mathrm{~mm}$ de largo, negruzcos, hispídulos sobre las 5 costillas, vilano en forma de copa de menos de $0.5 \mathrm{~mm}$ de largo, coronada por 15 a 25 lóbulos escuamiformes.

Tipo: México, Oaxaca, cerro Pelón, municipio de Comaltepec, distrito de Ixtlán, alt. 2950 m, matorral de ericáceas, 26.II.1972, J. Rzedowski 28838 (IEB).

O. juarezensis indudablemente es cercano a O. arbutifolius (H.B.K.) A. Gray, así como a $O$. oaxacanus Blake y difiere de ambos en el follaje concentrado en la 


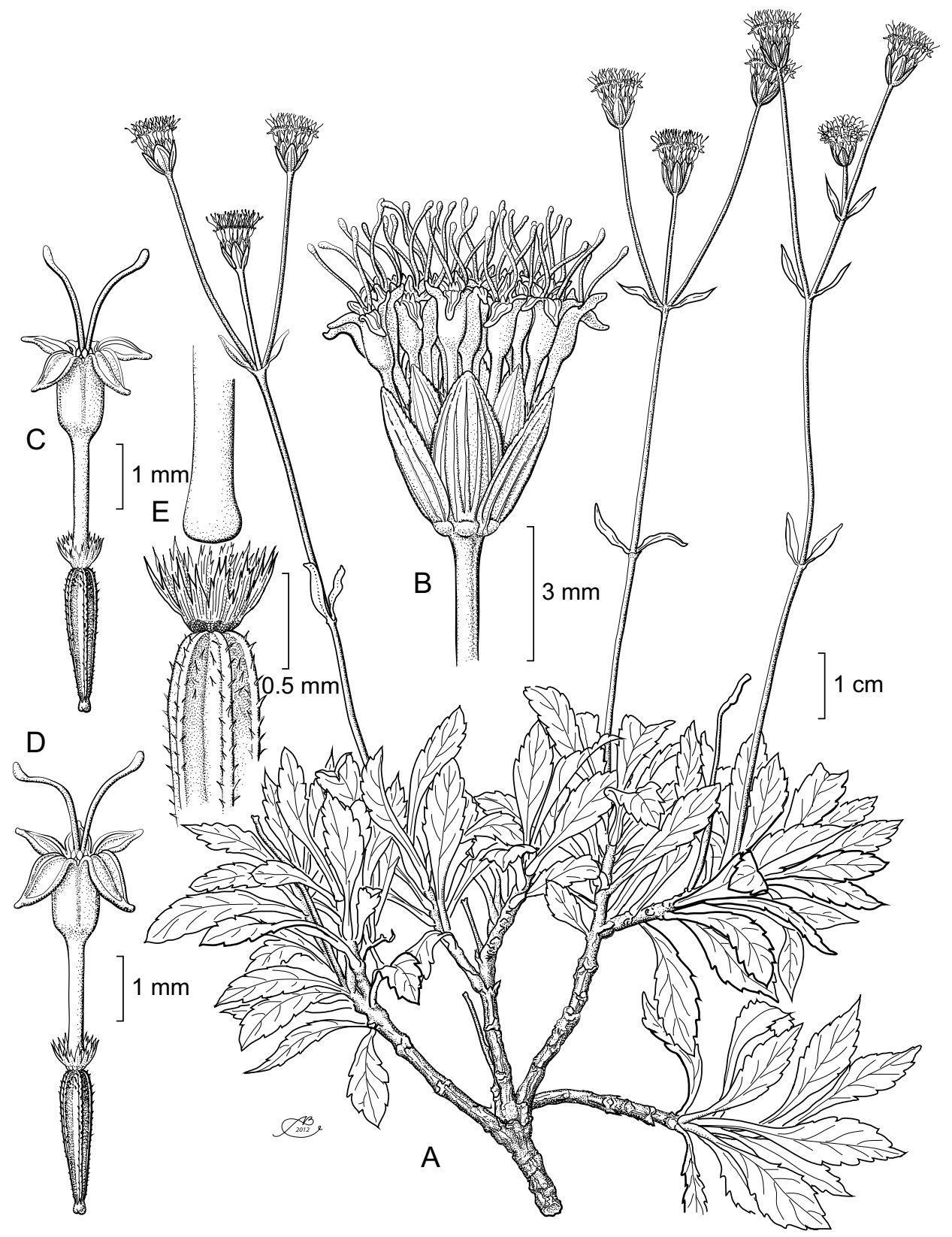

Fig. 2. Oxylobus juarezensis Rzed. \& Calderón. A. aspecto general de la planta; B. cabezuela; C. flor del centro del disco; D. flor de la periferie del disco; E. detalle del aquenio, vilano y base de la corola. Ilustrado por Alfonso Barbosa. 
parte basal de la planta, así como en ser totalmente glabro y en el margen de las hojas que a menudo es dentado.

En el cerro Pelón la especie nueva convive con el mucho más abundante $O$. oaxacanus, con el que comparte el carácter de cabezuelas con relativamente pocas (15 o menos) flores. Sin embargo, las dos plantas difieren entre sí muy notablemente no sólo en el porte general, sino también en la forma de la inflorescencia que es abierta en la primera y compacta en la segunda.

El carácter de las flores periféricas algo diferentes de las del centro del disco no es exclusivo de esta especie, pues lo comparten también otros representantes del género, como cabe observar en las figs. 1, 3 y 5 del trabajo de Turner y Kerr (1985).

El epíteto del taxon que se describe hace alusión a la comarca en que se ha encontrado, conocida comúnmente como la Sierra de Juárez, nombre que a su vez se ha originado a partir del hecho de que Benito Juárez (1806-1862), ameritado presidente de México e impulsor de relevantes reformas a la vida política del país, nació en la población de Guelatao, ubicada en el seno de este macizo montañoso.

Es de trascendencia comentar que casi todas las especies conocidas de Oxylobus se han colectado en la parte alta de la Sierra de Juárez, por lo que ésta constituye indudablemente el principal centro de diversidad del género. En conexión con lo anterior sería de mucho interés la realización de un profundo estudio en el área, tendiente a definir los procesos evolutivos que se han realizado y se siguen realizando allí y sobre todo a perfeccionar el esquema taxonómico de Oxylobus.

\section{AGRADECIMIENTOS}

Los autores agradecen a Billie L. Turner sus comentarios constructivos al manuscrito.

\section{LITERATURA CITADA}

Shinners, L. H. 1946. Revision of the genus Kuhnia. Wrightia 1: 122-144.

Shinners, L. H. 1971. Kuhnia L. transferred to Brickellia Ell. (Compositae). Sida 4: 274. 
Turner, B. L. 1989. An overview of the Brickellia (Kuhnia) eupatorioides (Asteraceae, Eupatorieae) complex. Phytologia 67: 121-131.

Turner, B. L. y K. M. Kerr. 1985. Revision of the genus Oxylobus (Asteraceae-Eupatorieae). Pl. Syst. Evol. 151: 73-87. 